\title{
Kinetics of circulating endothelial progenitor cells in patients undergoing carotid artery surgery
}

\author{
This article was published in the following Dove Press journal: \\ Therapeutics and Clinical Risk Management \\ 14 December 2016 \\ Number of times this article has been viewed
}

\author{
Günay Kalender' \\ Angela Kornberger ${ }^{2}$ \\ Milan Lisy' \\ Andres Beiras-Fernandez ${ }^{2}$ \\ Ulrich A Stock ${ }^{2}$ \\ 'Deparment of General, Thoracic and \\ Vascular Surgery, Hoechst Hospital, \\ ${ }^{2}$ Department of Thoracic and \\ Cardiovascular Surgery, University \\ Hospital Frankfurt, Frankfurt am Main, \\ Germany
}

\begin{abstract}
Aim: Endothelial progenitor cells (EPCs) are primitive cells found in the bone marrow and peripheral blood (PB). In particular, the potential of EPCs to differentiate into mature endothelial cells remains of high interest for clinical applications such as bio-functionalized patches for autologous seeding after implantation. The objective of this study was to determine EPCs' kinetics in patients undergoing carotid artery thromboendarterectomy (CTEA) and patch angioplasty.
\end{abstract}

Methods: Twenty CTEA patients were included (15 male, mean age 76 years). PB samples were taken at 1 day preoperatively, and at 1, 3, and 5 days postoperatively. Flow cytometric analysis was performed for CD34, CD133, KDR, and CD45. Expression of KDR, SDF-1 $\alpha$, and G-CSF was analyzed by means of enzyme-linked immunosorbent assay.

Results: Fluorescence-activated cell sorting analysis revealed $0.031 \% \pm 0.016 \%$ ( $\%$ of PB mononuclear cells) $\mathrm{KDR}+$ cells and $0.052 \% \pm 0.022 \% \mathrm{CD} 45-/ \mathrm{CD} 34+/ \mathrm{CD} 133+$ cells, preoperatively. A 33\% decrease of CD45-/CD34+/CD133+ cells was observed at day 1 after surgery. However, a relative number (compared to initial preoperative values) of CD45-/CD34+/CD133+ cells was found on day $3(82 \%)$ and on day 5 (94\%) postoperatively. More profound upregulated levels of CD45-CD34+/CD133+ cells were observed for diabetic (+47\% compared to nondiabetic) and male ( $+38 \%$ compared to female) patients. No significant postoperative time-dependent differences were found in numbers of KDR+ cells and the concentrations of the cytokines KDR and G-CSF. However, the SDF- $1 \alpha$ levels decreased significantly on day 1 postoperatively but returned to preoperative levels by day 3 .

Conclusion: CTEA results in short-term downregulation of circulating EPCs and SDF-1 $\alpha$ levels. Rapid return to baseline levels might indicate participation of EPCs in repair mechanisms following vascular injury.

Keywords: endothelial, progenitor, cells, carotid, artery, surgery

\section{Introduction}

Endothelial progenitor cells (EPCs) are predifferentiated adult stem cells with a potential to proliferate and differentiate into mature endothelial cells (ECs). ${ }^{1-3}$ In contrast to mature ECs, which are differentiated cells with low proliferative potential and limited ability to regenerate damaged endothelium, EPCs have a high proliferative potential, contribute to reendothelialization after vascular injury, and are involved in neovascularization of ischemic tissues. ${ }^{4-6}$ Levels of circulating EPCs are known to be reduced in patients with cardiovascular disease ${ }^{7}$ or patients at risk of cardiovascular disorder. ${ }^{8}$ EPC levels have also been shown to be predictive of mortality in patients with coronary artery disease. ${ }^{9,10}$ Recently, EPCs have gained more interest for potential clinical applications as in vivo endothelialization of biologic and prosthetic materials. ${ }^{11}$ As a proof of concept, we have shown in previous works the capacity of
Correspondence: Andres Beiras-

Fernandez

Department of Thoracic and Cardiovascular Surgery, University Hospital Frankfurt, Theodor-Stern-Kai 7, 60590 Frankfurt am Main, Germany Tel +496963016112

Fax +496963014391

Email andres.beiras@kgu.de 
bio-functionalized polytetrafluoroethylene material to couple murine EPC. ${ }^{12}$

The objective of this paper was to study the effect of a defined surgical vascular trauma by means of carotid artery thromboendarterectomy (CTEA) on the levels and kinetics of peripheral circulating putative EPCs and concentrations of the cytokines SDF-1 $\alpha, \mathrm{KDR}$, and G-CSF.

\section{Materials and methods}

\section{Patients, surgical procedure, and}

\section{sample acquisition}

Patients with asymptomatic internal carotid artery (ICA) stenosis ( $>70 \%$ as determined by ultrasonography) and scheduled for surgery were enrolled in the study (Table 1). The study was approved by the Ethics Review Board of the University of Tuebingen Medical Faculty (number: 403/2007BO2). Informed consent was obtained from each participant. Twenty patients were enrolled (75\% male; age range 54-87 years). Healthy male Caucasian volunteers ( $n=18$, age range $23-43$ years) served as controls.

Patients received general anesthesia and were intubated receiving controlled ventilation. The main carotid artery (MCA), ICA, and external carotid artery were exposed. Six cubic centimeters of blood was collected, and a polyethyleneterephthalate patch (Bard, Murray Hills, NJ, USA) was preclotted for 10 minutes. Following systemic heparinization (100 IU/kg), the external carotid artery was cross clamped. The MCA and ICA were temporarily occluded, and the MCA was longitudinally incised with extension into the ICA behind the stenosis. A $12 \mathrm{~F}$ shunt was inserted, and brain perfusion reestablished (mean occlusion time was $90 \pm 15$ s). An endarterectomy was performed, and the preclotted patch was

Table I Demographic data

\begin{tabular}{lll}
\hline Cardiovascular risk factors & $\mathbf{n}$ & $\%$ \\
\hline Age (years) & 1 & \\
$<60$ & 2 & 5 \\
$6 I-70$ & 10 & 10 \\
$7 I-80$ & 7 & 50 \\
$>80$ & $15 / 5$ & 35 \\
Sex (male/female) & 20 & $75 / 25$ \\
Arterial hypertension & 8 & 100 \\
Diabetes (IDDM) & 12 & 40 \\
Smoking & 5 & 60 \\
Actively & 7 & 25 \\
Previously & & 35 \\
BMI (kg/m²) & 1 & \\
$<20$ & 10 & 5 \\
$20-30$ & 9 & 50 \\
$>30$ & & 45 \\
\hline A6brevation
\end{tabular}

Abbreviations: IDDM, insulin-dependent diabetes mellitus; BMI, body mass index. implanted using running 6-0 polypropylene sutures (Ethicon, Somerville, NJ, USA). Immediately prior to completion of the suture, the shunt was removed with concordant reocclusion of the MCA and ICA. The suture was completed, the patch area thoroughly deaired, and perfusion reestablished (mean occlusion time was $80 \pm 15 \mathrm{~s}$ ). Heparin was not reversed.

Steady-state venous peripheral blood (PB) samples $(15 \mathrm{~mL})$ were collected at 1 day preoperatively and at 1,3 , and 5 days postoperatively.

\section{PBMCs isolation}

PB mononuclear cells (PBMCs) were isolated by Histopaque1077 density gradient centrifugation $(800 \times g, 30$ minutes) following the manufacturer's instructions. Briefly, isolated PBMCs were washed once in phosphate-buffered saline (PBS), centrifuged (400×g, 7 minutes), and resuspended in $1 \mathrm{~mL}$ PBS supplemented with $1 \mathrm{mM}$ ethylenediaminetetraacetic acid and $2 \%$ human serum albumin.

\section{Flow cytometry (fluorescence- activated cell sorting)}

EPCs were analyzed for surface marker expression with an LSRII instrument (BD Biosciences, San Jose, CA, USA). Flow cytometric analysis was performed for CD34, CD133, VEGF-R2, and CD144. CD45 was added to lower the detection threshold and to distinguish between hematopoietic and endothelial lineages.

Flow cytometry was performed to test for reactivity with the following antihuman monoclonal antibodies: APCVEGF-R2 (KDR, \#FAB357A; R\&D Systems Inc., Minneapolis, MN, USA), FITC-CD34 (\#130-081-001; Miltenyi Biotec, Bergisch Gladbach, Germany), PE-CD133 (\#130090-853; Miltenyi Biotec), AF700-CD144 (\#56-1449-73; eBioscience, San Diego, CA, USA), and CD45 (\#PB-222T100; Exbio, Vestec, Czech). A second VEGF-R2 antibody (\#6251; Santa Cruz Biotechnology, Santa Cruz, CA, USA) labeled with an AF647-Zenon ${ }^{\circledR}$ Mouse IgG Labeling Kit (\#Z25008; Thermo Fisher Scientific, Waltham, MA, USA), following manufacturer's instructions, was used.

Briefly, PBMCs (15-20×10\%/15 mL blood) were washed in PBS, centrifuged ( 7 minutes, $400 \times g$ ), and resuspended in $1 \mathrm{~mL}$ PBS (supplemented with $1 \mathrm{mM}$ ethylenediaminetetraacetic acid and $2 \%$ human serum albumin). Cells $\left(2 \times 10^{6}\right)$ were stained with fluorochrome-labeled antibodies in a total volume of $100 \mu \mathrm{L}$ for 30 minutes on ice in darkness. Appropriate negative control isotype-matched antibodies (BD [Franklin Lakes, NJ, USA] and Miltenyi) were used in the same concentration as tested antibodies. Additionally, fluorescence-minus-one negative controls were measured. 
For compensation of signal overlaps in multichannel fluorescence-assisted cell sorting measurements, CD3 antibodies (Exbio) were used with corresponding chromophores. Dead and apoptotic cells were excluded by staining with 7AAD (559925; 1:250; BD). Data from $10^{6}$ events were acquired, and were analyzed with BD FACSDiva (version 6.0; BD Biosciences) software.

\section{Enzyme-linked immunosorbent assays}

Approximately, $2 \mathrm{~mL}$ of blood plasma aliquots was taken from each Histopaque separation. VEGF-R2, SDF-1 $\alpha$, and G-CSF concentrations were determined using cytokine-specific enzyme-linked immunosorbent assays according to the manufacturer's instructions (Quantikine; R\&D Systems Inc.).

\section{Statistical analysis}

For statistical analysis, we conducted one-way betweensubjects analysis of variance for the four time points, with day of measurement as the independent variable and percentage of EPCs or cytokine concentration as dependent variables. Statistical analysis was performed using PASW Statistics software (version 18.0.0; SPSS Inc., Chicago, IL, USA). $P$-values $<0.05$ were considered statistically significant.

\section{Results}

\section{Flow cytometric analysis}

EPCs were analyzed following the gating strategy depicted in Figure 1D. 7AAD+ cells were excluded as nonvital or
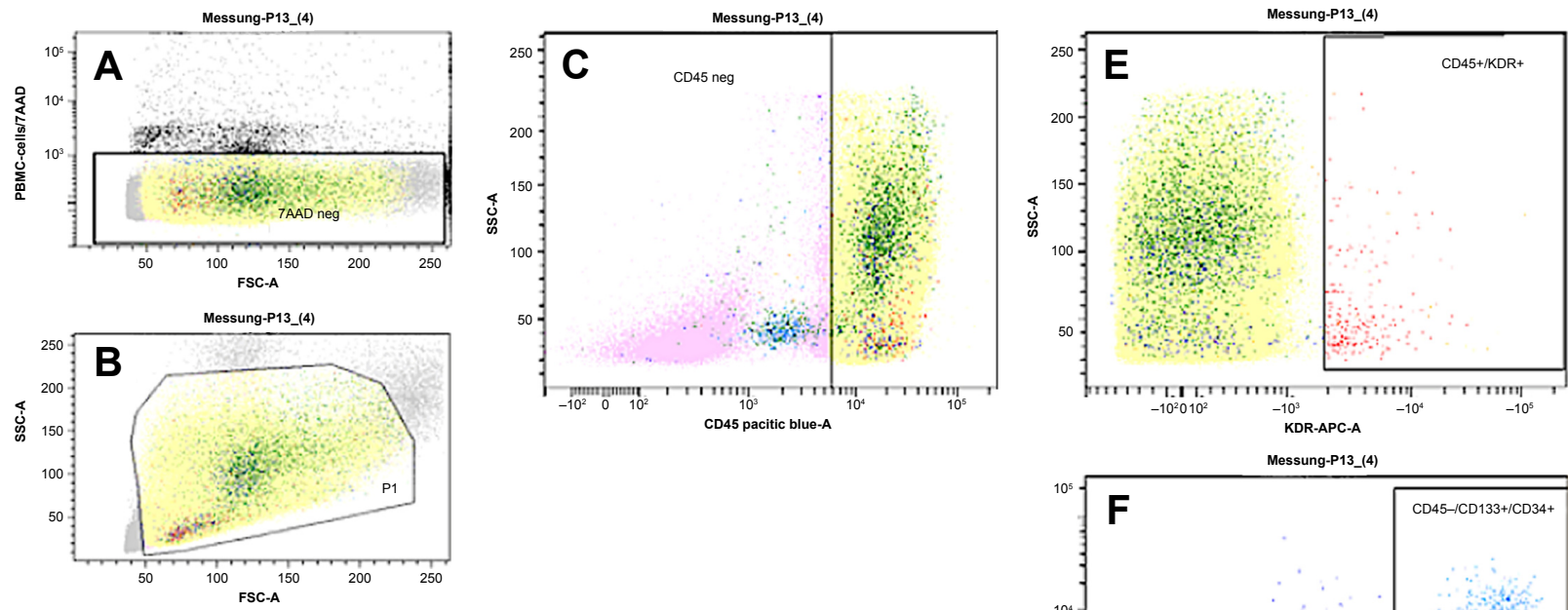

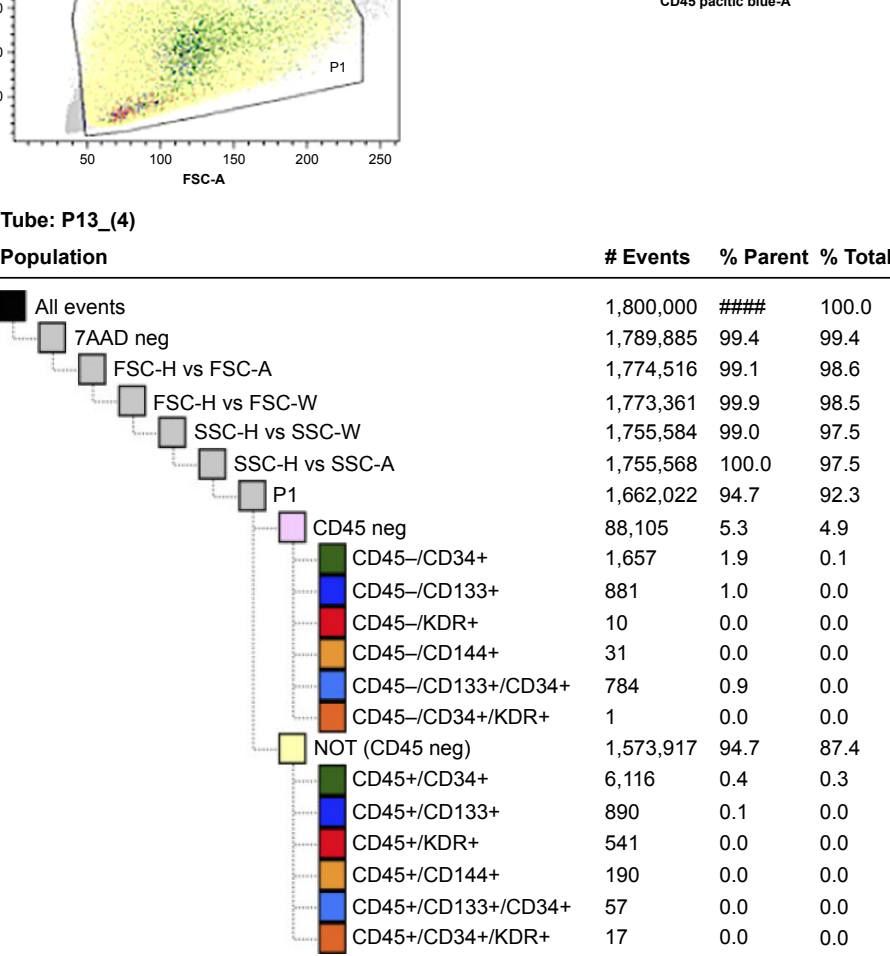

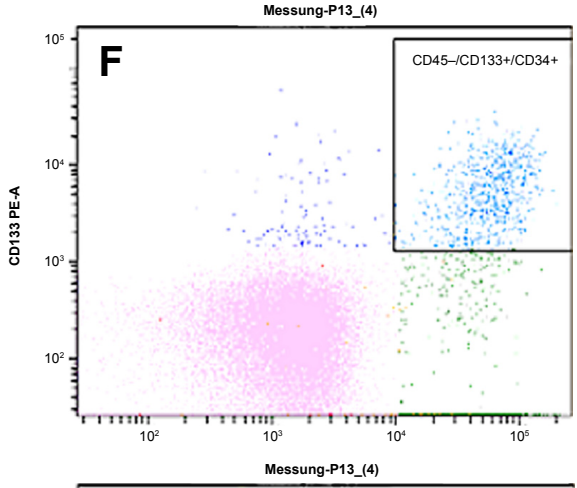

Tube: P13_(4)

D Population

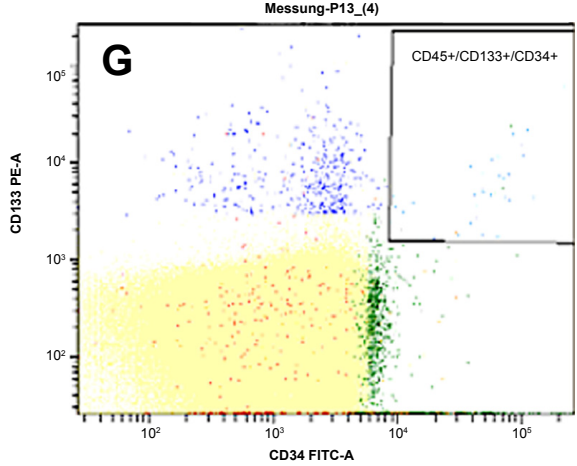

Figure I FACS gating strategy for quantification of PBMCs with EPC character.

Notes: Living (7AAD negative, A) and single cells (normal front- and side-scatter signals) within the monocyte and lymphocyte PBMC population (B) were analyzed. Additionally, CD45 was used as a preselecting marker (C) to exclude cells of the hematopoietic pathway from the analysis. According to the FACS gating strategy (D), two populations were found within the PBMCs to fit into the current model of EPC expression patterns: a CD45+/KDR+ population (E, early EPCs) and a CD45-/CD34+/ CDI33+ population ( $\mathbf{F}$ and $\mathbf{G}$, matured EPCs).

Abbreviations: FACS, fluorescence-activated cell sorting; PBMCs, peripheral blood mononuclear cells; EPCs, endothelial progenitor cells. 
damaged cells (Figure 1A). Doublet cells and agglomerates were excluded according to their increased side and front scatter. A gate including the monocyte and lymphocyte populations was selected for further analysis (Figure 1B). CD45, a typical marker of the hematopoietic lineage, was chosen as a preselection marker (Figure 1C; pink: CD45-; yellow: CD45+). The analysis was focused on the potential EPC markers: KDR, CD34, and CD133.

Of the gated PBMCs, $0.031 \% \pm 0.016 \%$ stained positive for KDR. Of the KDR+ PBMCs, 79\% were CD45+ $(0.027 \% \pm 0016 \%$; Figure $1 \mathrm{E}-\mathrm{G}) . \mathrm{KDR}+$ cells were mostly found within the lymphocyte population revealing a small cell size with low granularity. Only negligible $(0.001 \%)$ amounts of KDR+ cells were positive for CD34. Parallel experiments with the two different KDR antibodies revealed comparable results.

Of the analyzed PBMCs, $0.209 \% \pm 0.143 \%$ stained positive for CD34, and $0.108 \% \pm 0.042 \%$ for CD 133 .
Two CD34+ subpopulations were detected when CD45 was involved. The same was found for CD133. Sixty-nine percent of CD34+ and 52\% of CD133+ cells were negative for CD45. A strong overlap between both populations was observed $(0.059 \% \pm 0.025 \%)$, but this overlap was almost exclusively found within the CD45- population. Size and granularity of the CD45-/CD34+, CD45-/CD133+, as well as the CD45-/ CD34+/CD133+ PBMCs were comparable to the small nongranular lymphocyte population, whereas CD45+ counterparts were partially located within the monocytes.

CD144 was found in $0.033 \% \pm 0.028 \%$ of PBMCs, but this endothelial marker was not coexpressed with any other tested marker. Summarized, flow cytometric analysis revealed two potential EPC populations: $0.052 \% \pm 0.022 \%$ ( $\%$ of PBMCs) of CD45-/CD34+/CD133+ cells and $0.034 \% \pm 0.024 \%$ of $\mathrm{KDR}+$ cells.

Quantitative variation in the expression profiles of the putative EPC populations due to the surgical trauma was

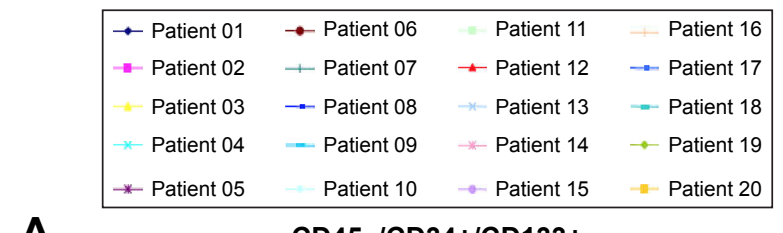

A

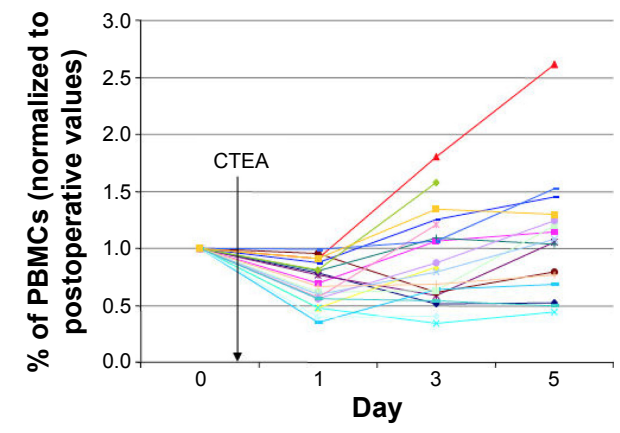

C

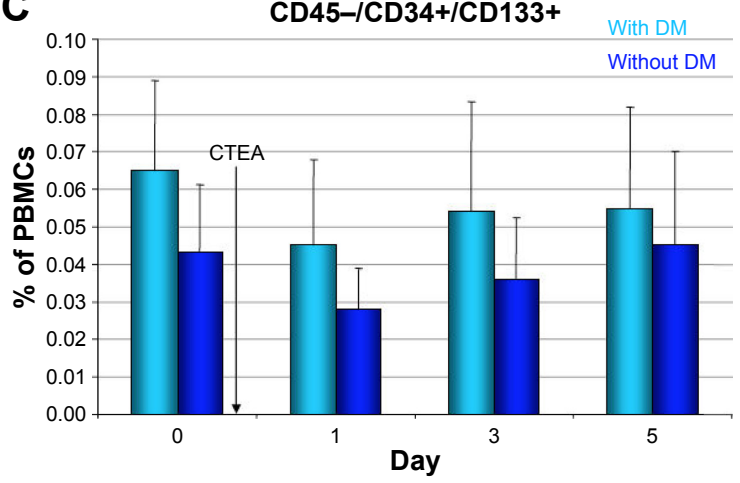

B

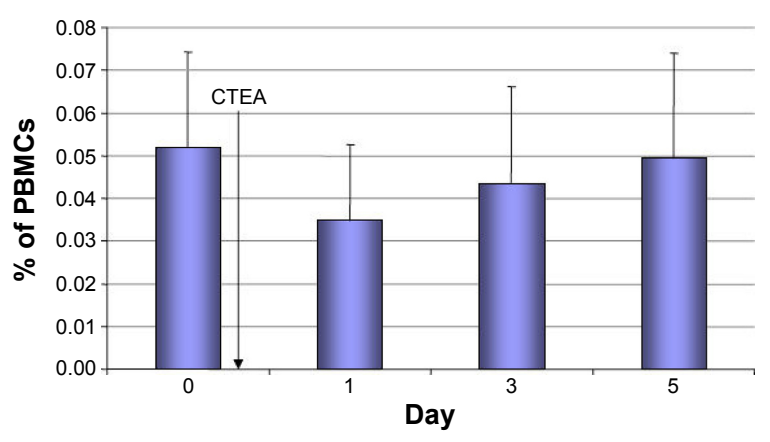

D

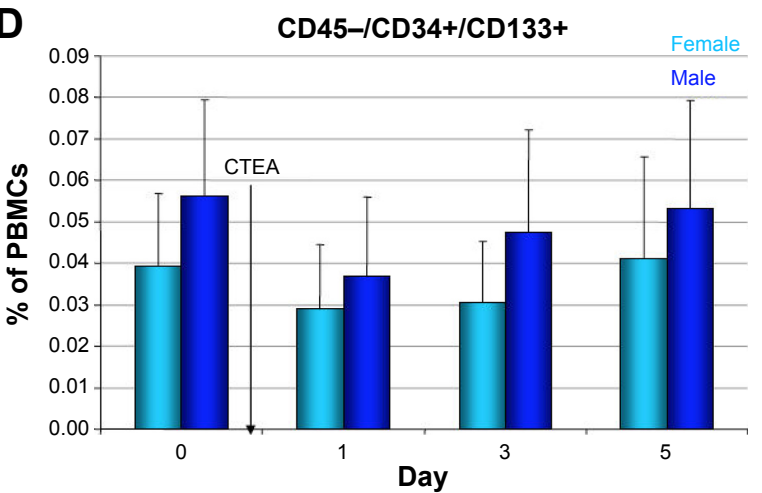

Figure 2 Progression of values of CD45-/CD34+/CDI33+ cells after surgical impact.

Notes: On the day after surgery, a 33\% decrease of CD45-/CD34+/CDI33+ cells was observed; however, levels returned to $82 \%$ on days 3 and $94 \%$ on day 5 postoperatively. Relative cell numbers are depicted for each patient $(\mathbf{A})$ and as daily mean \pm standard deviation (B). The number of CD45-/CD34+/CDI33+ PBMCs were found to be increased for diabetes mellitus patients (light blue) compared to nondiabetic patients (C), and the values were also increased for male patients compared to females (D). Abbreviations: PBMCs, peripheral blood mononuclear cells; CTEA, carotid artery thromboendarterectomy; DM, diabetes mellitus. 

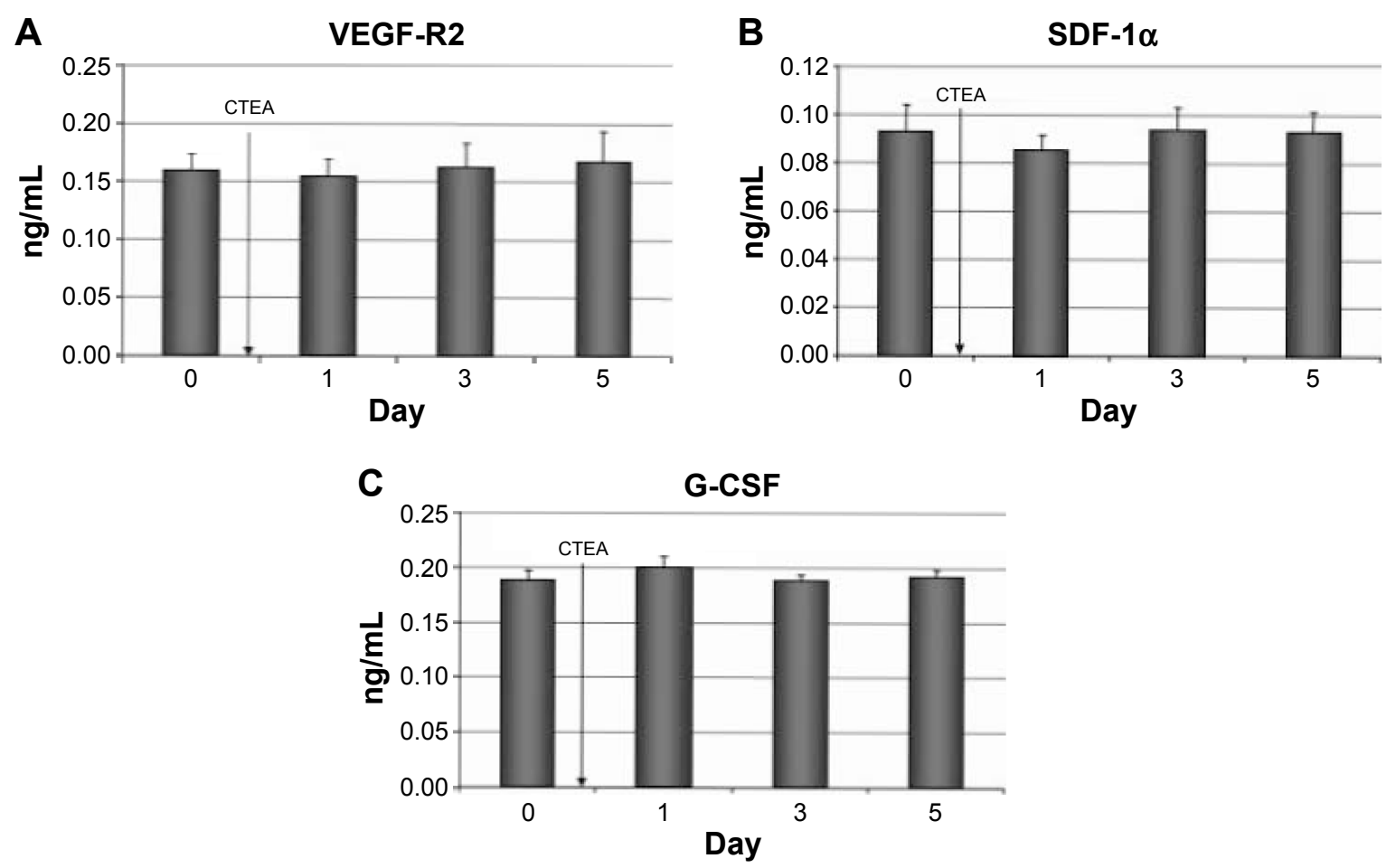

Figure 3 ELISAs for cytokines associated to EPC activation and angiogenesis.

Notes: No significant effect of CTEA was found for human soluble VEGF-R2 (A). Human SDF-I $\alpha$ (B) decreased significantly by $8 \%$, and human G-CSF (C) increased by $6 \%$. Both values returned to initial values on days 3 and 5 .

Abbreviations: ELISAs, enzyme-linked immunosorbent assays; EPC, endothelial progenitor cell; CTEA, carotid artery thromboendarterectomy

determined (Figure 2A). Whereas no significant changes were found for VEGF-R2+/CD45+ cells $(F(3,72)=1.184$; $P=0.322)$ after surgery, significant changes were detected for CD45-CD34+/CD133+ cells $(F(3,72)=4.994 ; P=0.003)$. $t$-Tests revealed a significant $33 \%$ drop of CD $45-/ C D 34+/$ CD133+ cells on the day after CTEA $(t(38)=7.299$; $P<0.001$ ). However, a relative number (compared to initial preoperative values) of CD45-/CD34+/CD133+ cells was found on day $3(82 \% ; t(38)=-2,081 ; P=0.047)$ and on day 5 $(94 \% ; t(34)=-1,201 ; P=0.240)$ postoperatively (Figure $2 \mathrm{~B})$. A more profound upregulation of CD45-/CD34+/CD133+ cells was observed for diabetics $(+44 \%$, day 0$)$ when values of labeled cells were compared to nondiabetic patients (Figure 2C). Male patients had 38\% more CD45-/CD34+/ CD133+ cells (day 0) than females (Figure 2D).

\section{Plasma cytokines}

Concentrations of cytokines, involved in the EPC activation, were measured in plasma aliquots (Figure 3 ) by means of enzyme-linked immunosorbent assay. Analysis of variance tests revealed a significant effect of the vascular impact on concentrations of SDF-1 $\alpha(F(3,36)=3.450 ; P=0.027)$. For SDF- $1 \alpha, t$-tests revealed a significant $8.4 \%$ decrease directly after surgery $(t(18)=2.671 ; P=0.026)$ and a significant recovery from day 1 to day $3(t(18)=-2.325 ; P=0.033)$. No significant effect was found for concentrations of VEGF-R2 $(F(3,36)=1.357 ; P=0.271)$. A slight preoperative increase of G-CSF concentrations was measured, but differences were proved not to be significant $(F(3,36)=2.409 ; P=0.083)$.

\section{Discussion}

Since discovery in 1997, an increasing interest has been devoted to the study of EPCs, a subtype of immature cells involved in endothelial repair and neo-angiogenesis, and cells that play a comprehensive role in tissue homeostasis.

Many studies have addressed the identity of EPCs, but their exact definition is still in ongoing discussion. ${ }^{13-16}$ Most of the surface marker combinations used in flow cytometry studies included the marker CD34 and VEGF-R2 because initial studies in the EPC field reported that CD34+ and VEGF-R2+ cells purified from various sources (umbilical cord blood, PB, bone marrow) and distinct chronic cardiovascular diseases (dilative cardiomyopathy ${ }^{17}$ ) were able to generate ECs in vitro. ${ }^{1,3,18}$ CD34+/CD133+/VEGF-R2+ cells are widely accepted to identify true circulating EPCs, but yet these cells were never directly tested to generate new ECs in vitro or in vivo. ${ }^{13,19}$ In this study, two putative EPC populations were detected: VEGF-R2+ cells and a 
CD34+/CD133+ population. Additionally, we prescreened with the common leukocyte antigen CD45, to lower the background threshold by excluding cells of hematopoietic CD45+ fraction. ${ }^{14}$ Positive events were calculated as percent of living singlet cells within the monocyte/lymphocyte fraction of Histopaque-1077 density gradient-separated PBMCs. Of the PBMCs, $0.034 \%$ were VEGF-R2+, but most of these cells (79\%) were found in the CD45+ fraction; $0.052 \%$ were marked positive for CD34+/CD133+, and this population was found in the CD45- fraction. A strong overlap of CD34+ and CD133+ cells was observed, but no overlap was seen for CD34+ and VEGF-R2 markers. ${ }^{14}$

Impacts on numbers of EPCs in general are manifold. Increasing numbers of EPCs are described to correlate with physiological variations such as raised estrogenic levels ${ }^{14}$ or physical exercises. ${ }^{20}$ They are known to be impaired in patients suffering from coronary artery disease (termed as CD34+/VEGF-R2+), those who smoke, ${ }^{21}$ or those suffering from hyperlipidemia ${ }^{22}$ compared to healthy volunteers. Even small variations such as the human circadian rhythm impact levels of circulating EPCs. ${ }^{23}$

In our study, a significant (33\% of PBMCs) decrease of CD45-/CD34+/CD133+ cells was observed on day 1 after surgery. However, a relative number (compared to initial preoperative values) of CD45-CD34+/CD133+ cells was found on day $3(82 \%)$ and on day $5(94 \%)$ postoperatively. A potential mechanism of the observed decrease in EPCs might be an active participation in reendothelialization of the traumatized lumen of the carotid artery and patch surface. The surgical impact itself did not show any effect on the level of VEGF-R2+ cells. Furthermore, increased levels of CD45-/CD34+/CD133+ cells were found in diabetic patients compared to nondiabetics. This observation is confirmed by a recent publication indicating an impaired correlation between diabetes and EPC levels. ${ }^{24-26}$ However, these groups focused on functional assay such as endothelial tube formation ${ }^{25,26}$ or proliferative capacity ${ }^{27}$ rather than expressed surface markers.

Recent works have shown that specific cytokines are involved in EPC mobilization. G-CSF is a well-known cytokine used extensively in clinical settings to mobilize hematopoietic stem cells. G-CSF is produced by activated monocytes/macrophages, fibroblasts, ECs, and bone marrow stromal cells. An increase was observed on day 1 after CTEA in our study, but levels were decreasing to initial values on days 3 and 5. We did not measure any effect on VEGF concentration. The SDF- $1 \alpha$ levels decreased significantly by $8 \%$ on day 1 postoperatively but returned to preoperative levels by day 5 . Similar results in terms of preoperative cytokine trends were reported from Roberts et al. ${ }^{28}$ They measured these cytokines post- and preoperatively in patients undergoing cardiac surgery. Mieno et $\mathrm{al}^{29}$ published data describing increased SDF-1 $\alpha$ levels 4 hours after coronary artery bypass grafting which returned to initial values by day 4 preoperatively. We hypothesize that a vascular trauma such as a CTEA triggers a G-CSF release resulting in an increased EPC mobilization. The exact mechanism for such release remains to be clarified. It is possible that other cytokines, such as SDF-1 $\alpha$, are implicated prior to G-CSF release. Smaller time intervals between preoperative measurements could reveal a more detailed time course analysis of the exact mechanism of EPC mobilization.

\section{Conclusion}

Clear limitations of the study are relative small sample size indicating the need for further in-depth investigations to elaborate potential applications such as in vivo EPC capture (adhesion) and subsequent differentiation of biomaterials.

\section{Acknowledgment}

The authors gratefully acknowledge the German Research Foundation (Deutsche Forschungsgemeinschaft) for generous funding (Sto 359/7-1).

\section{Disclosure}

The authors report no conflicts of interest in this work.

\section{References}

1. Asahara T, Murohara T, Sullivan A, et al. Isolation of putative progenitor endothelial cells for angiogenesis. Science. 1997;275(5302): 964-967.

2. Hristov M, Erl W, Weber PC. Endothelial progenitor cells: mobilization, differentiation, and homing. Arterioscler Thromb Vasc Biol. 2003; 23(7):1185-1189.

3. Shi Q, Rafii S, Wu MH, et al. Evidence for circulating bone marrowderived endothelial cells. Blood. 1998;92(2):362-367.

4. Asahara T, Takahashi T, Masuda H, et al. VEGF contributes to postnatal neovascularization by mobilizing bone marrow-derived endothelial progenitor cells. EMBO J. 1999;18(14):3964-3972.

5. Urbich C, Dimmeler S. Endothelial progenitor cells: characterization and role in vascular biology. Circ Res. 2004;95(4):343-353.

6. Roberts N, Jahangiri M, Xu Q. Progenitor cells in vascular disease. J Cell Mol Med. 2005;9(3):583-591.

7. Vasa M, Fichtlscherer S, Aicher A, et al. Number and migratory activity of circulating endothelial progenitor cells inversely correlate with risk factors for coronary artery disease. Circ Res. 2001;89(1):E1-E7.

8. Hill M, Cibula D, Havlikova H, et al. Circulating levels of pregnanolone isomers during the third trimester of human pregnancy. J Steroid Biochem Mol Biol. 2007;105(1):166-175.

9. Werner N, Kosiol S, Schiegl T, et al. Circulating endothelial progenitor cells and cardiovascular outcomes. N Engl J Med. 2005;353: 999-1007. 
10. Schmidt-Lucke C, Rossig L, Fichtlscherer S, et al. Reduced number of circulating endothelial progenitor cells predicts future cardiovascular events: proof of concept for the clinical importance of endogenous vascular repair. Circulation. 2005;111(22):2981-2987.

11. Schleicher M, Wendel HP, Fritze O, Stock UA. In vivo tissue engineering of heart valves: evolution of a novel concept. Regen Med. 2009;4(4): 613-619.

12. Schleicher M, Hansmann J, Elkin B, et al. Oligonucleotide and parylene surface coating of polystyrene and ePTFE for improved endothelial cell attachment and hemocompatibility. Int J Biomater. 2012; 2012:397813.

13. Timmermans F, Plum J, Yoder MC, Ingram DA, Vandekerckhove B, Case J. Endothelial progenitor cells: identity defined? J Cell Mol Med. 2009;13(1):87-102.

14. Fadini GP, Baesso I, Albiero M, Sartore S, Agostini C, Avogaro A. Technical notes on endothelial progenitor cells: ways to escape from the knowledge plateau. Atherosclerosis. 2008;197(2):496-503.

15. Kienstra KA, Hirschi KK. Vascular progenitor cell mobilization. Methods Mol Biol. 2012;904:155-164.

16. Yoder MC. Endothelial progenitor cell: a blood cell by many other names may serve similar functions. J Mol Med (Berl). 2013;91(3): 285-295.

17. Roura S, Gálvez-Montón C, Fernández MA, Lupón J, Bayes-Genis A. Circulating endothelial progenitor cells: potential biomarkers for idiopathic dilated cardiomyopathy. J Cardiovasc Transl Res. 2016;9(1): 80-84.

18. Bompais H, Chagraoui J, Canron X, et al. Human endothelial cells derived from circulating progenitors display specific functional properties compared with mature vessel wall endothelial cells. Blood. 2004 103(7):2577-2584.

19. Peichev M, Naiyer AJ, Pereira D, et al. Expression of VEGFR-2 and AC133 by circulating human CD34(+) cells identifies a population of functional endothelial precursors. Blood. 2000;95(3):952-958.
20. Adams V, Lenk K, Linke A, et al. Increase of circulating endothelial progenitor cells in patients with coronary artery disease after exercise-induced ischemia. Arterioscler Thromb Vasc Biol. 2004;24(4): 684-690.

21. Kondo T, Hayashi M, Takeshita K, et al. Smoking cessation rapidly increases circulating progenitor cells in peripheral blood in chronic smokers. Arterioscler Thromb Vasc Biol. 2004;24(8):1442-1447.

22. Li TB, Zhang JJ, Liu B, et al. Involvement of NADPH oxidases and non-muscle myosin light chain in senescence of endothelial progenitor cells in hyperlipidemia. Naunyn Schmiedebergs Arch Pharmacol. 2016 389(3):289-302.

23. Thomas HE, Redgrave R, Cunnington MS, Avery P, Keavney BD, Arthur HM. Circulating endothelial progenitor cells exhibit diurnal variation. Arterioscler Thromb Vasc Biol. 2008;28:e21-e22.

24. Fadini GP, Sartore S, Schiavon M, et al. Diabetes impairs progenitor cell mobilisation after hindlimb ischaemia-reperfusion injury in rats Diabetologia. 2006;49(12):3075-3084.

25. Loomans CJ, de Koning EJ, Staal FJ, et al. Endothelial progenitor cell dysfunction: a novel concept in the pathogenesis of vascular complications of type 1 diabetes. Diabetes. 2004;53(1):195-199.

26. Hill JM, Zalos G, Halcox JP, et al. Circulating endothelial progenitor cells, vascular function, and cardiovascular risk. $N$ Engl J Med. 2003; 348(7):593-600.

27. Tepper OM, Galiano RD, Capla JM, et al. Human endothelial progenitor cells from type II diabetics exhibit impaired proliferation, adhesion, and incorporation into vascular structures. Circulation. 2002;106(22):2781-2786.

28. Roberts N, Xiao Q, Weir G, Xu Q, Jahangiri M. Endothelial progenitor cells are mobilized after cardiac surgery. Ann Thorac Surg. 2007; 83(2):598-605.

29. Mieno S, Ramlawi B, Boodwani M, et al. Role of stromal-derived factor1alpha in the induction of circulating CD34+CXCR4+ progenitor cells after cardiac surgery. Circulation. 2006;4;114(Suppl 1):I186-I192.
Therapeutics and Clinical Risk Management

\section{Publish your work in this journal}

Therapeutics and Clinical Risk Management is an international, peerreviewed journal of clinical therapeutics and risk management, focusing on concise rapid reporting of clinical studies in all therapeutic areas, outcomes, safety, and programs for the effective, safe, and sustained use of medicines. This journal is indexed on PubMed Central, CAS,

\section{Dovepress}

EMBase, Scopus and the Elsevier Bibliographic databases. The manuscript management system is completely online and includes a very quick and fair peer-review system, which is all easy to use. Visit http://www.dovepress.com/testimonials.php to read real quotes from published authors. 